\title{
Standard Model Higgs boson production in association with a top anti-top pair at NLO with parton showering
}

\author{
M. V. Garzelli ${ }^{1}$, A. $\operatorname{Kardos}^{1}$, C. G. Papadopoulos ${ }^{2}$ and Z. Trócsányi ${ }^{1,3}$ \\ ${ }^{1}$ Institute of Physics, University of Debrecen, H-4010 Debrecen P.O.Box 105, Hungary \\ ${ }^{2}$ NCSR Demokritos, Institute of Nuclear Physics, GR-15310 Athens, Greece \\ ${ }^{3}$ Institute of Nuclear Research of the Hungarian Academy of Sciences, Hungary
}

\begin{abstract}
We present predictions for the production cross section of a Standard Model Higgs boson in association with a t $\overline{\mathrm{t}}$ pair at next-to-leading order accuracy using matrix elements obtained from the HELAC-Oneloop package. The NLO prediction was interfaced to the PYTHIA and HERWIG shower Monte Carlo programs with the help of POWHEG-Box, allowing for decays of massive particles, showering and hadronization, thus leading to final results at the hadron level.
\end{abstract}

PACS 12.38.-t - Quantum ChromoDynamics

PACS 13.87.-a - Jets in large- $Q^{2}$ scatterings

PACS 14.65.Ha - Top quarks

PACS 14.80.Bn - Standard Model Higgs boson 


\section{Introduction}

In recent years a plethora of NLO QCD calculations have been presented in the literature. High-energy experiments, notably at the LHC, have and will be benefited by the progress in our computational ability to deal with higher order corrections to scattering amplitudes with many partons involved. In order though to get the optimum benefit and to produce predictions that can be directly compared to experimental data at the hadron level, a matching with parton showers and hadronization is ultimately inevitable.

According to the literature, two methods exist that deal with the matching of parton showers (PS) to matrix element (ME) calculations at NLO accuracy, MC@NLO [1, 2] and POWHEG [3, 4]. At the same time, several computational frameworks for NLO QCD calculations have emerged. In constructing a general interface of PS to ME computations with NLO accuracy, we have chosen to combine the POWHEG and HELAC-NLO [5] approach, respectively. The first application of our project was presented in Ref. [6].

In this letter we continue our investigation and validation of such an interface by studying Higgs boson production in association with a t $\bar{t}$ pair. There are several reasons to perform this work. On the phenomenological side, there is still interest to use this channel to search for Higgs boson in the low mass regime [7]. On the theoretical side, the appearance of recently developed tools as implemented in aMCONLO [8], also used in the analysis of Higgs boson production in association with a t $\bar{t}$ pair, offers a unique opportunity for cross-checking and validating our computations and the corresponding computer programs.

\section{Method}

We performed our calculations using the POWHEG-Box [9], a flexible computer framework implementing the POWHEG method, already used in the study of different processes also by other authors (among the others, the most recent applications include Ref. [10, 11, 12, 13]). In this work the following ingredients were requested and provided to POWHEG-Box as input:

- The flavor structures of the Born $(\mathrm{gg} \rightarrow \mathrm{Ht} \overline{\mathrm{t}}, \mathrm{q} \overline{\mathrm{q}} \rightarrow \mathrm{Ht} \overline{\mathrm{t}}, \overline{\mathrm{q}} \mathrm{q} \rightarrow \mathrm{Ht} \overline{\mathrm{t}})$ and real radiation emission $(\mathrm{q} \overline{\mathrm{q}} \rightarrow \mathrm{Ht} \overline{\mathrm{t}} \mathrm{g}, \mathrm{gg} \rightarrow \mathrm{Ht} \overline{\mathrm{tg}}, \overline{\mathrm{q}} \mathrm{g} \rightarrow \mathrm{Ht} \overline{\mathrm{t}} \overline{\mathrm{q}}, \mathrm{g} \overline{\mathrm{q}} \rightarrow \mathrm{Ht} \overline{\mathrm{t}} \overline{\mathrm{q}}, \overline{\mathrm{q}} \mathrm{q} \rightarrow \mathrm{Ht} \overline{\mathrm{tg}} \mathrm{g}, \mathrm{qg} \rightarrow \mathrm{Ht} \overline{\mathrm{t}} \mathrm{q}, \mathrm{gq} \rightarrow$ Htt̄q) subprocesses $(\mathrm{q} \in\{\mathrm{u}, \mathrm{d}, \mathrm{c}, \mathrm{s}, \mathrm{b}\})$ were specified.

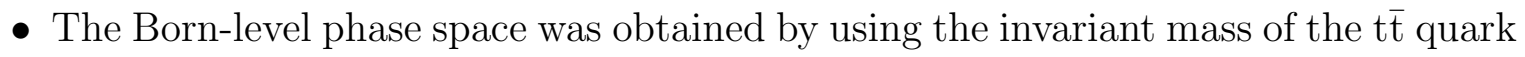
pair and four angles.

- Squared ME's with all incoming momenta for the Born and the real-emission processes were built using amplitudes computed by HELAC-Oneloop [14], on the basis of skeleton structures generated by this same program and HELAC-PHEGAS [15], respectively. The ME's in the physical channels were obtained by crossing.

- Color structures for building the color-correlated Born amplitudes were taken from HELAC-Dipoles [16].

- Spin-correlated Born amplitudes were projected from the helicity basis to the Lorentz one by using the polarization vectors. 
- HELAC-Oneloop was also used for computing the finite part of the virtual correction contributions in dimensional regularization, on the basis of the OPP method [17] complemented by Feynman Rules for the computation of the QCD $R_{2}$ Rational terms [18].

With this input POWHEG-Box was used to generate events at the Born level plus first radiation, stored in Les Houches Event Files (LHEF) [19]. Then, one can choose any shower Monte Carlo (SMC) program for generating events with hadrons, just by showering the previous ones and applying hadronization and decay models (it is worth mentioning that POWHEG-Box was written in the effort of being shower detail independent). We worked with both the PYTHIA [20, 21] and HERWIG [22, 23] SMC generators.

In the POWHEG-Box the first emission is the hardest one measured by transverse momentum which is also the ordering variable in PYTHIA. However, if the ordering variable in the shower is different from this one, such as in HERWIG, the hardest emission can be different from the first one. In such cases, in order to face this issue, HERWIG is constrained to discard shower evolutions (vetoed shower) with larger transverse momentum in all splittings occuring after the first emission, that is computed by POWHEG-Box . In addition, a truncated shower, simulating wide-angle soft emission before the hardest emission, is in principle also needed for POWHEG matching [3], but its effect turned out to be small [24]. As there is no implementation of truncated shower in HERWIG using external LHEF, the effect of the truncated shower is absent from our predictions.

\section{Checks}

The Born and the real ME's were tested in randomly chosen phase-space points against results from HELAC-PHEGAS . The consistency among real emission, Born, color-correlated and spin-correlated ME's was tested in randomly chosen real-emission phase-space regions, by examining the ratio between the real-emission amplitudes in soft and collinear limits and the corresponding subtraction terms, automatically computed by POWHEG-Box as approaching the related singularities.

The process presented here was studied extensively at the NLO accuracy in the literature [25, 26, 27, 28, 29, which enabled us to make detailed checks of our calculation.

\begin{tabular}{|c|c|c|c|}
\hline \hline & $m_{H}, \mathrm{GeV}$ & $\sigma_{\text {lit. }}^{\mathrm{NLO}}, \mathrm{fb}$ & $\sigma_{\mathrm{PH}}^{\mathrm{NLO}}, \mathrm{fb}$ \\
\hline \hline \multirow{2}{*}{ TeVatron } & 120 & $4.857(8)$ & $4.851(3)$ \\
& 140 & $2.925(4)$ & $2.918(2)$ \\
$(\sqrt{s}=2 \mathrm{TeV})$ & 160 & $1.806(2)$ & $1.797(2)$ \\
& 180 & $1.132(1)$ & $1.128(1)$ \\
\hline \multirow{2}{*}{$\mathrm{LHC}$} & 120 & $701.5(18)$ & $701.3(8)$ \\
& 140 & $452.3(12)$ & $452.8(5)$ \\
$(\sqrt{s}=14 \mathrm{TeV})$ & 160 & $305.6(8)$ & $305.0(4)$ \\
& 180 & $214.0(6)$ & $213.9(3)$ \\
\hline
\end{tabular}

Table 1: Comparison of the NLO cross sections by POWHEG-HELAC $(\mathrm{PH})$ to the predictions of Ref. [26] (lit) at the default scale $\mu_{R}=\mu_{F}=\mu_{0}$. 

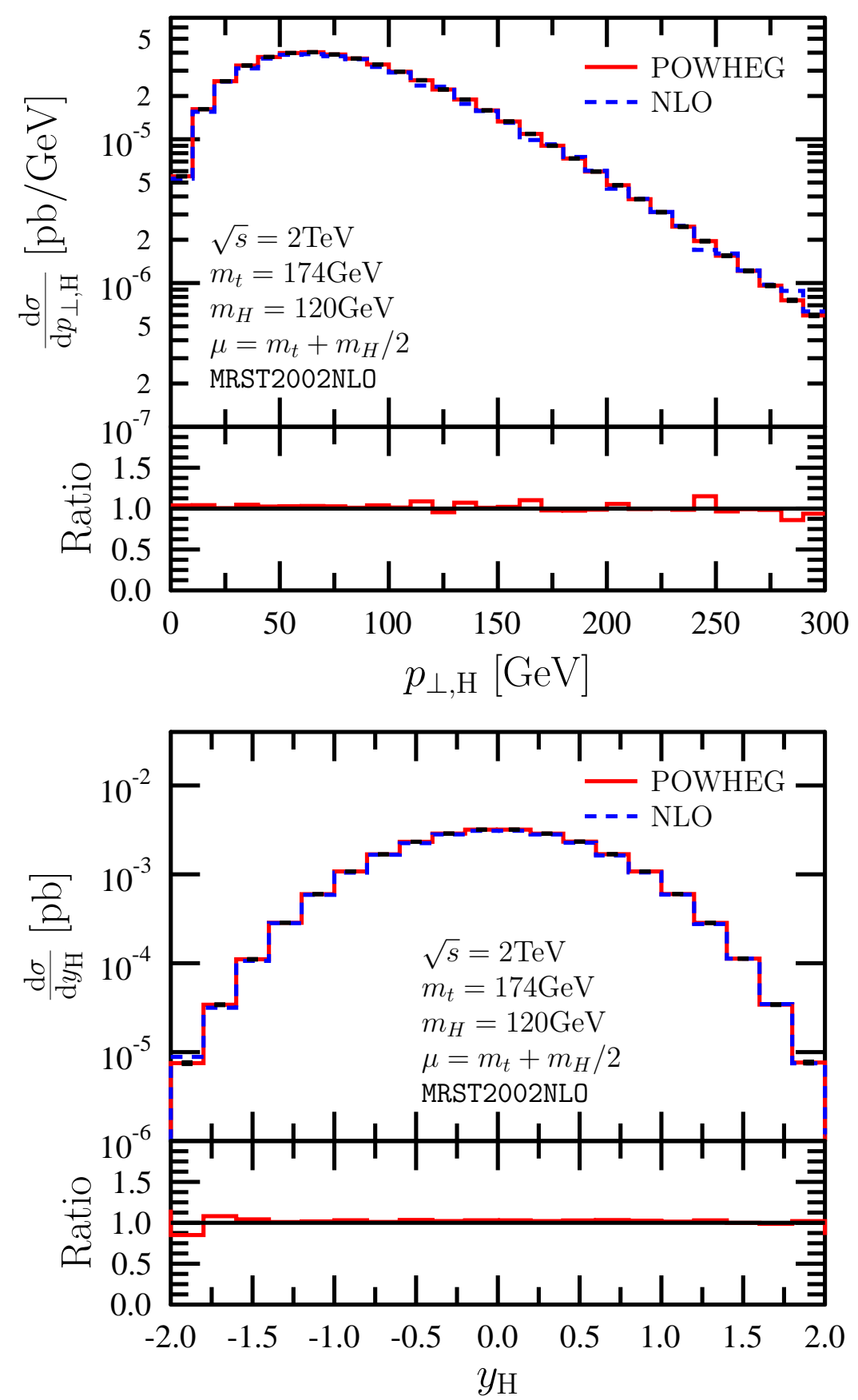

Figure 1: Transverse momentum and rapidity distributions of the Higgs boson at the Tevatron from our calculation by means of POWHEG-HELAC (POWHEG) and from Ref. [26] (pure NLO). The lower panels show the ratio of the results (POWHEG/NLO).

As for the virtual contribution, we compared our amplitudes to the predictions of MADLOOP using the input parameters and phase space point specified in Ref. [30] for different renormalization scale choices [31, and in all cases we found agreement up to 5-6 digits. 
We then compared the cross-sections at the NLO accuracy to the predictions of Ref. [26]. At this purpose, we used the MRST2002nlo 32] PDF set, provided by LHAPDF [33], with a 2-loop running $\alpha_{s}, 5$ light flavours and $\Lambda_{5}^{\overline{\mathrm{MS}}}=239 \mathrm{MeV}$, and the following parameters: $m_{H}=120 \mathrm{GeV}, m_{t}=174 \mathrm{GeV}, G_{F}=1.16639 \cdot 10^{-5} \mathrm{GeV}^{-2}$. The renormalization and factorization scales were chosen equal to $\mu$, where $\mu$ was varied in the $\mu_{0} / 4-$ $4 \mu_{0}$ interval, with default scale being $\mu_{0}=m_{t}+m_{H} / 2$. The calculations were performed at the planned LHC energy of $\sqrt{s}=14 \mathrm{TeV}$. The results of this comparison are presented in Table 1, showing agreement. We also made a comparison to the predictions of Ref. [29], using the CTEQ5M PDF set from LHAPDF, a 2-loop running $\alpha_{s}$ with $\alpha_{s}\left(M_{Z}\right)=0.118, m_{t}$ and $G_{F}$ as given above, and again found agreement within their quoted uncertainty.

Finally, we also compared differential distributions from events already including first radiation emission with the NLO predictions of Ref [26]. We found agreement for all distributions presented in that paper. As examples, we show in Fig. 1 the transverse momentum and rapidity distributions of the Higgs boson in the Tevatron setup 1 .

\section{The effect of the shower}

In interfacing NLO calculations to SMC programs we mainly aim at estimating the effects of showers and hadronization, therefore, we analyzed the events at two different stages of evolution:

Decay: starting from the events collected in LHEF produced by POWHEG-HELAC, we just included on-shell decays of t-quarks and the Higgs boson, as implemented in PYTHIA, and further decays of their decay products, like charged leptons (the $\tau$ is assumed to be unstable), $W$ and $Z$, turning off any shower and hadronization effect.

Full SMC: decays, showering evolution and hadronization have been included in our simulations, using both PYTHIA and HERWIG.

In both SMC setup muons (default in PYTHIA) and neutral pions were assumed as stable particles. All other particles and hadrons were allowed to be stable or to decay according to the default implementation of each SMC. Quark and Higgs masses, as well as $W, Z$ masses and total decay widths, were tuned to the same values in PYTHIA and HERWIG. On the other hand, each of the two codes was allowed to compute autonomously partial branching fractions in different decay channels for all unstable particles and hadrons. Multiparticle interaction effects were neglected (default in HERWIG ). Additionally, the intrinsic $p_{T}$ spreading of valence partons in incoming hadrons in HERWIG was assumed to be $2.5 \mathrm{GeV}$. Considering this setup, we found agreement between PYTHIA and HERWIG predictions within $5 \%$, except in bins where the statistics is very small. Beside the conceptual differences in the parton shower and hadronization algorithms between the two SMC generators, written on the basis of different theoretical ideas ( $p_{\perp}$ vs. angular ordering, string model vs. cluster hadronization and preconfinement), a possible origin of this overall small discrepancy is the absence of the truncated shower in the HERWIG

\footnotetext{
${ }^{1}$ Note that these comparisons refer to distributions without normalization, as opposed to the plots in the original publication.
} 
prediction. Unfortunately, however, as already mentioned, we cannot check the last point within the POWHEG-Box framework. In any case, the modest entity of the discrepancy means that the effect of the truncated shower, not included in our analysis, is small. Further explanations of small discrepancies can also be related to the different decay channel scheme and branching fractions in the two SMC codes.

In our computation, we adopted the following parameters: $\sqrt{s}=7 \mathrm{TeV}$, CTEQ6.6M PDF set from LHAPDF, with a 2-loop running $\alpha_{s}, 5$ light flavours and $\Lambda_{5}^{\overline{\mathrm{MS}}}=226 \mathrm{MeV}$, $m_{t}=172 \mathrm{GeV}, m_{H}=120 \mathrm{GeV}, G_{F}=1.16639 \cdot 10^{-5} \mathrm{GeV}^{-2}$. The renormalization and factorization scales were chosen equal to $\mu_{0}=m_{t}+m_{H} / 2$. We decided to switch on all possible decay channels of the Higgs boson, implemented in the SMC programs. ${ }^{2}$ We used the last version of the SMC codes: PYTHIA 6.425 and HERWIG 6.520.

We studied the effect of the full SMC by comparing distributions at the decay and SMC level. As the number of particles is very different at the end of the two stages, we first made such a comparison without any selection cut, in order to avoid the introduction of any bias. This way the cross-section at all levels is indeed exactly the same. We found $\sigma_{\text {POWHEG-HELAC }}=\sigma_{\text {POWHEG-HELAC+DECAY }}=\sigma_{\text {POWHEG-HELAC }+ \text { SMC }}=95.872 \pm 0.007 \mathrm{fb}$ (here and in all following $\sigma$ predictions the quoted uncertainties are the statistical ones only). As an illustrative example, we present the distributions of the transverse momentum and rapidity of the hardest jet, $p_{\perp, j_{1}}$ and $y_{j_{1}}$, in Fig.2 and Fig.3 respectively. The jets are reconstructed through the anti $-k_{\perp}$ algorithm with $R=0.5$, by using Fast Jet 2.4.3 [34]. One can observe a rather significant softening in the transverse momentum spectrum as going from results at the decay level to full SMC ones. On the other hand, the effect of the shower on the rapidity of the hardest jet is almost negligible and rather homogeneous. We distinguished several classes of jets including final state emissions, according to their origin: jets that can be traced back to (i) first radiation emissions, (ii) the decay products of the Higgs boson, (iii) the decay products of the top and antitop quarks, (iv) a mixing of the previous ones. In particular, main contributions to the $p_{\perp, j_{1}}$ spectrum shown in Fig. 2 are due to jets of the (iv) and (iii) class. As for the $y_{j_{1}}$ distribution, the tails are dominated by jets of the (iv), (i) and (iii) classes.

In Ref. 30 the invariant mass, $m_{B B}$, and the separation in the rapidity-azimuthalangle plane of the two hardest lowest-lying B-hadrons, $\Delta R_{B B}$, were studied, by choosing a dynamical scale for the generation of the hard-scattering events and by taking into account only the $\mathrm{H} \rightarrow \mathrm{b} \overline{\mathrm{b}}$ decay channel. Besides computing all $p_{T}$ distributions already presented in that paper, always finding agreement, we also computed the aforementioned B-hadron distributions reproducing the same simulation setup and without applying any cut, considering only the $\mathrm{H} \rightarrow$ b $\bar{b}$ decay channel, as well as all channels. In the former, we found agreement with the predictions of Ref. [30]. The effect of the remaining channels, not studied in that work, produces an increase in the region below $80 \mathrm{GeV}$ in the $m_{B B}$ spectrum and only for large $\Delta R_{B B}$, as shown in Figs. 4 and 5 . Pairs of B-hadrons both including quarks that can be traced back to Higgs decays only populate the region of the $m_{B B}$ spectrum below $m_{H}$. The region above $m_{H}$ is instead dominated by pairs with at least one B-hadron that can be traced back to a (anti)top decay.

The effects of the decay and shower also depend on the selection cuts. While the typical selection cuts include both leptons and hadrons, we started with a restricted

\footnotetext{
${ }^{2}$ In PYTHIA there are two more decay channels than in HERWIG, and partial decay fractions in each leptonic, bosonic and partonic channel differ in the two codes.
} 


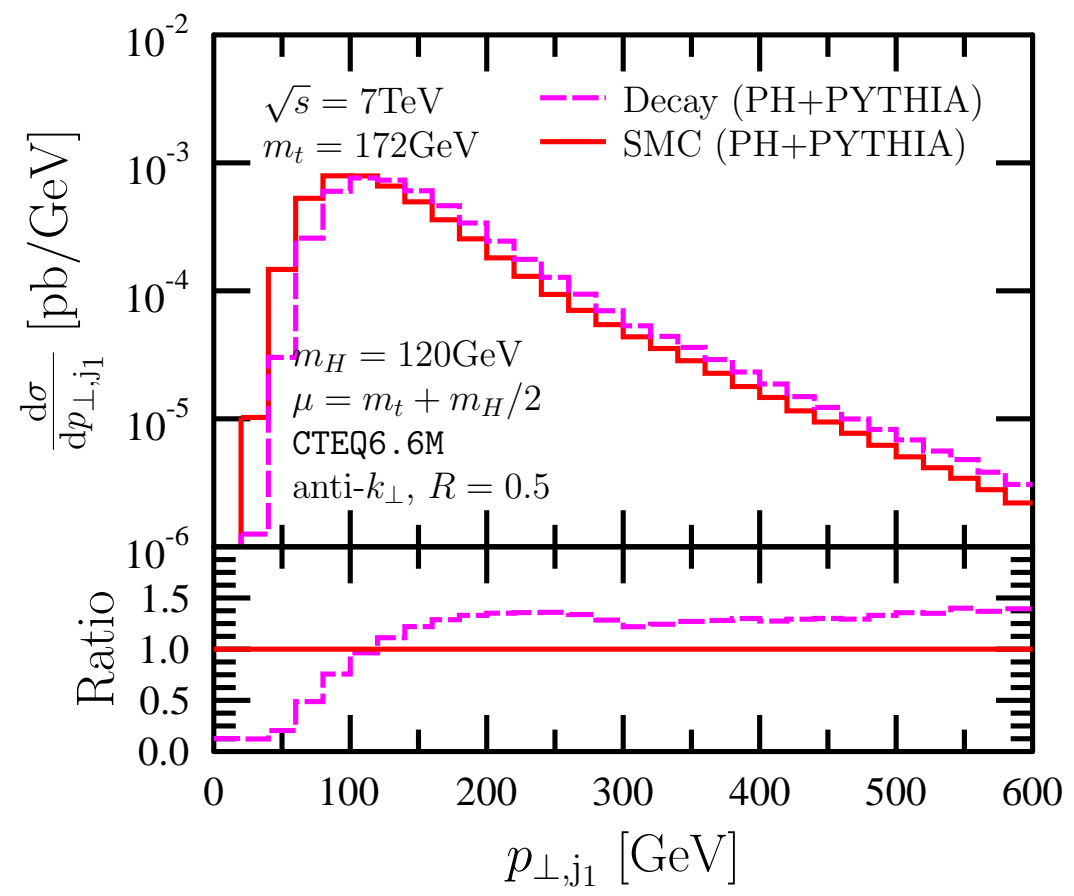

Figure 2: Transverse momentum distribution of the hardest jet. The results at the decay level (dashed line) are compared to the ones at the SMC level (solid line), obtained by interfacing POWHEG-HELAC to PYTHIA. No selection cuts were applied.

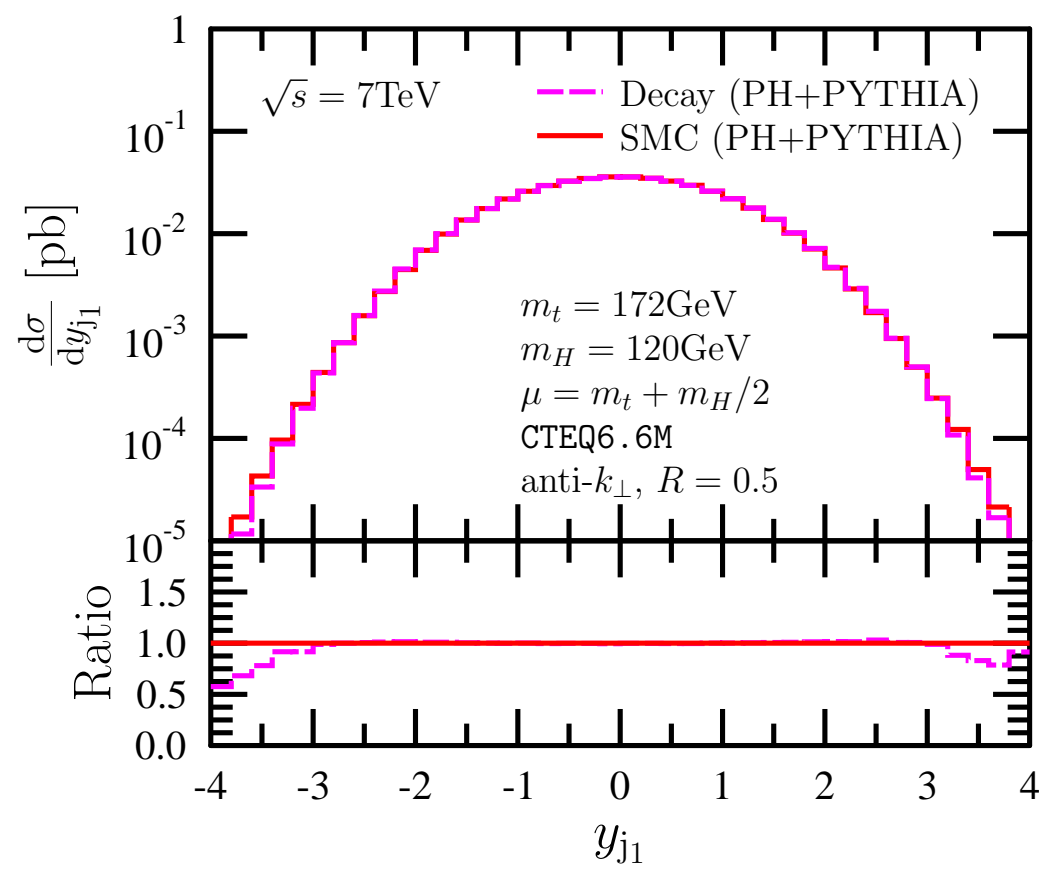

Figure 3: The same as Fig. 2, as for the rapidity of the hardest jet. 


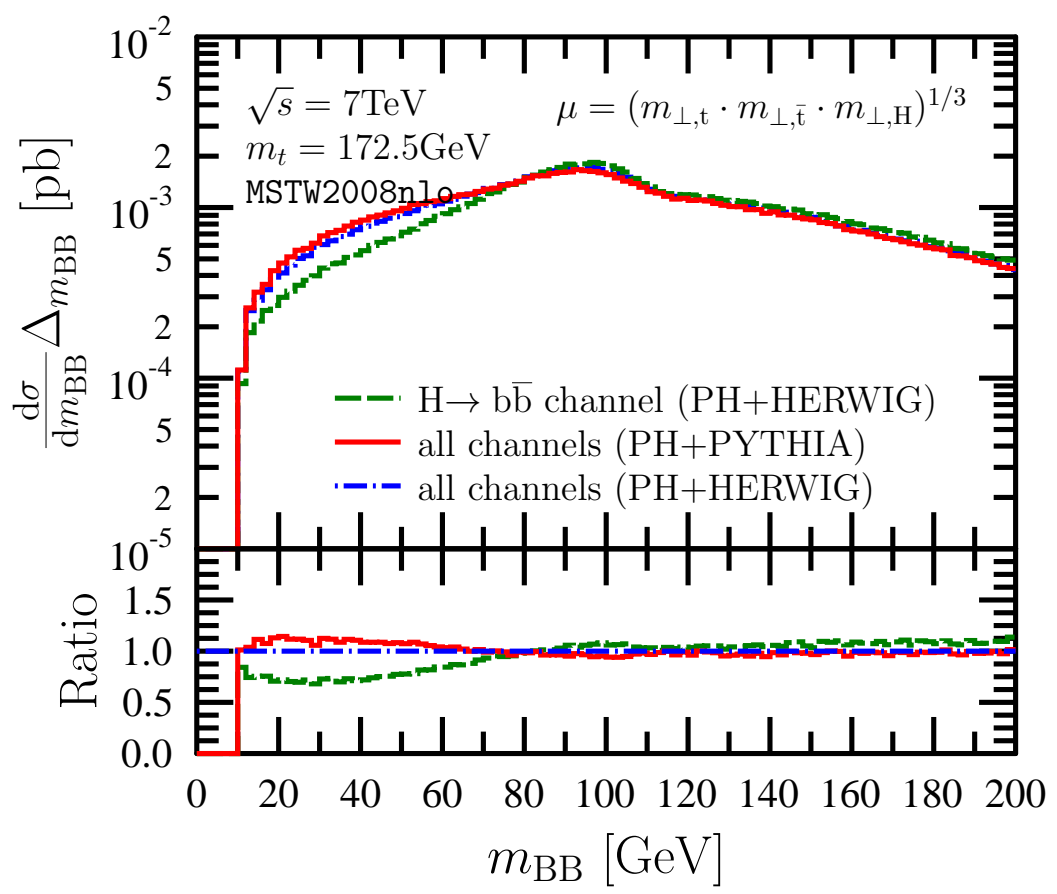

Figure 4: Invariant mass distribution of the two hardest lowest-lying B hadrons at the SMC level. These results are presented in sigma per bin, just to allow an easier comparison with the results of Ref. [30], having been obtained in the same setup, without applying any cut. The effects of including all $\mathrm{H}$ decay channels, with respect to the case of a single $\mathrm{H} \rightarrow \mathrm{b} \overline{\mathrm{b}}$ channel (dashed line), were computed by interfacing POWHEG-HELAC to both PYTHIA (solid line) and HERWIG (dash-dotted line).

set, not involving any leptonic cut, in particular as for the number of leptons. This is motivated by the fact that this number can be quite different at the decay and shower level, as the shower produce many secondary leptons. Thus, coming back to our setup, we considered and implemented cuts only on jet variables: (i) $p_{\perp, \min }^{\mathrm{j}}=20 \mathrm{GeV}$ and (ii) $\left|y^{\mathrm{j}}\right| \leq 2.5$ for all jets, (iii) \#jets $\geq 4$ in each event. We show the distribution of the scalar sum of all transverse momenta in the event, $H_{\perp}$ in Fig.6. We can see that the spectrum becomes softer due to showering effects, with respect to the one computed at the decay level, as can be understood on the basis of PS physics. The same is true and even more evident if one singles out the hadronic component of $H_{\perp}$, as well (not shown). On the other hand, the effects of the shower on the (anti-)lepton transverse momentum, $p_{\perp}^{\ell}$, and the missing transverse momentum, $p_{\perp}^{\text {miss }}$, as shown in Figs. 7 and 8, are small and rather uniform, except for a significant increase for small values, which is due to secondary leptons produced in the shower. The cross-sections at the decay and at the SMC level, after the hadronic cuts listed above, amount to $\sigma_{\text {POWHEG-HELAC }+ \text { DECAY }}=92.29 \pm$ $0.01 \mathrm{fb}, \sigma_{\text {POWHEG-HELAC }+ \text { PYTHIA }}=90.46 \pm 0.01 \mathrm{fb}$ and $\sigma_{\text {POWHEG-HELAC }+ \text { HERWIG }}=90.99 \pm 0.01 \mathrm{fb}$. 


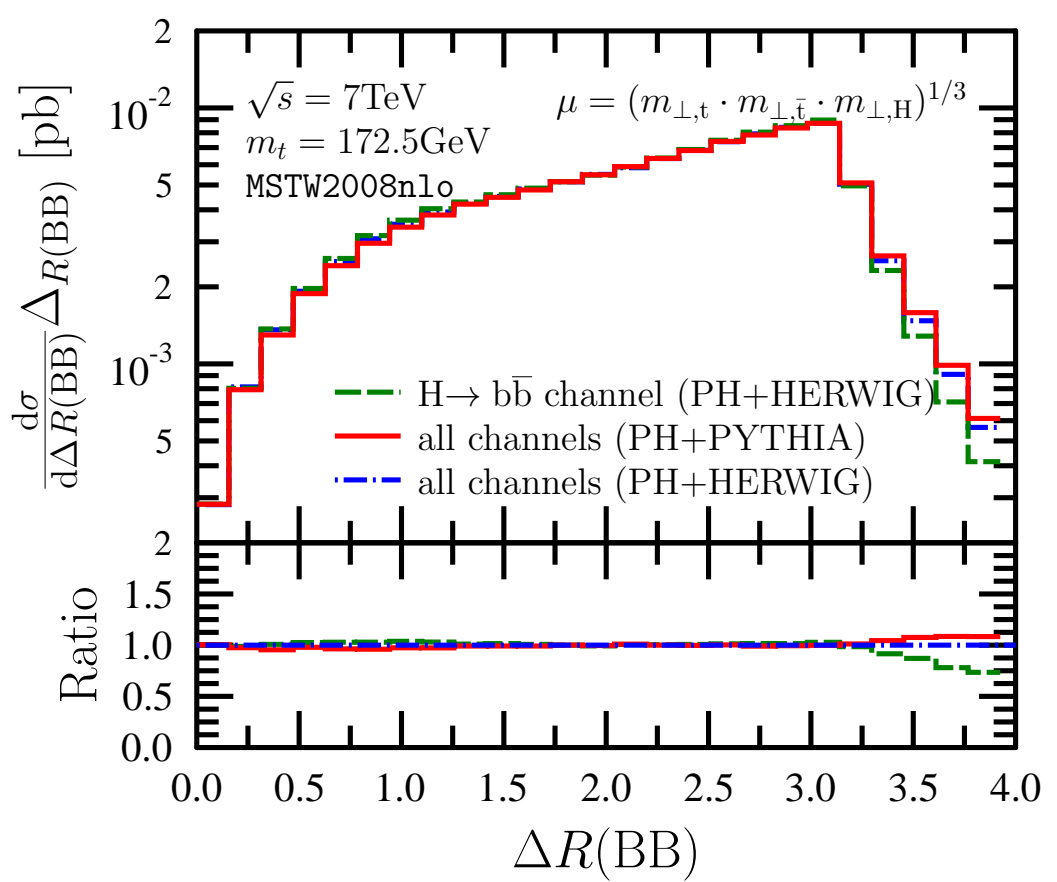

Figure 5: The same as Fig. 4, as for the azimuth-rapidity distance correlations of the two hardest lowest-lying B hadrons.

\section{$5 \quad$ Predictions}

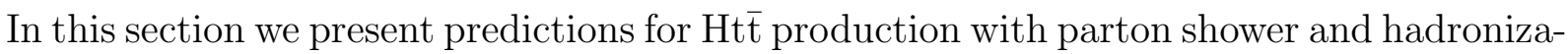
tion effects at LHC. In addition to the jet cuts (i-iii) mentioned above, we also applied selection cuts on the leptonic variables $3^{3}$ (iv) we focused on the dileptonic channel, asking for exactly one $\ell^{+}$and one $\ell^{-}$with $(\mathrm{v}) p_{\perp \text {, min }}^{\ell^{ \pm}}=20 \mathrm{GeV}$ and (vi) $\left|y^{\ell^{ \pm}}\right| \leq 2.5$, whereas the transverse missing energy of the event was constrained to (vii) $\mathbb{E}_{\perp, \min } \geq 30 \mathrm{GeV}$.

In Fig.9 we present the distribution of the invariant mass of all jet pairs. Here the effect of the shower is again quite significant. In particular, there is a small bump around the Higgs mass, as already noticed in the literature [35], visible in the data at the decay level, which is completely washed out when PS is included. The same is true for the invariant mass distribution of the two hardest jets (not shown).

The cross-section after cuts, at the SMC level, were found to be $\sigma_{\text {POWHEG-HELAC+PYTHIA }}=$ $5.376 \pm 0.010 \mathrm{fb}$ and $\sigma_{\text {POWHEG-HELAC }+ \text { HERWIG }}=5.521 \pm 0.011 \mathrm{fb}$.

One of the biggest differences in the results produced by PYTHIA and HERWIG interfaced to the POWHEG-Box noticed in Ref. [12] in the study of a different process was the observation that HERWIG gives rise to hard jets more central than PYTHIA. We observe the same trend in our results, but by far to a lesser extent. In particular, in the bins around zero rapidity the ratio between the rapidity distributions of POWHEG-HELAC + HERWIG and POWHEG-HELAC + PYTHIA found in our study amounts to maximum 1.05, both in case of the hardest and the second hardest jet. On the other hand, the agreement between the two SMC, as for the rapidity distributions of leptons and antileptons, was found to be

\footnotetext{
${ }^{3}$ Similar cuts are applied by the LHC experiments.
} 


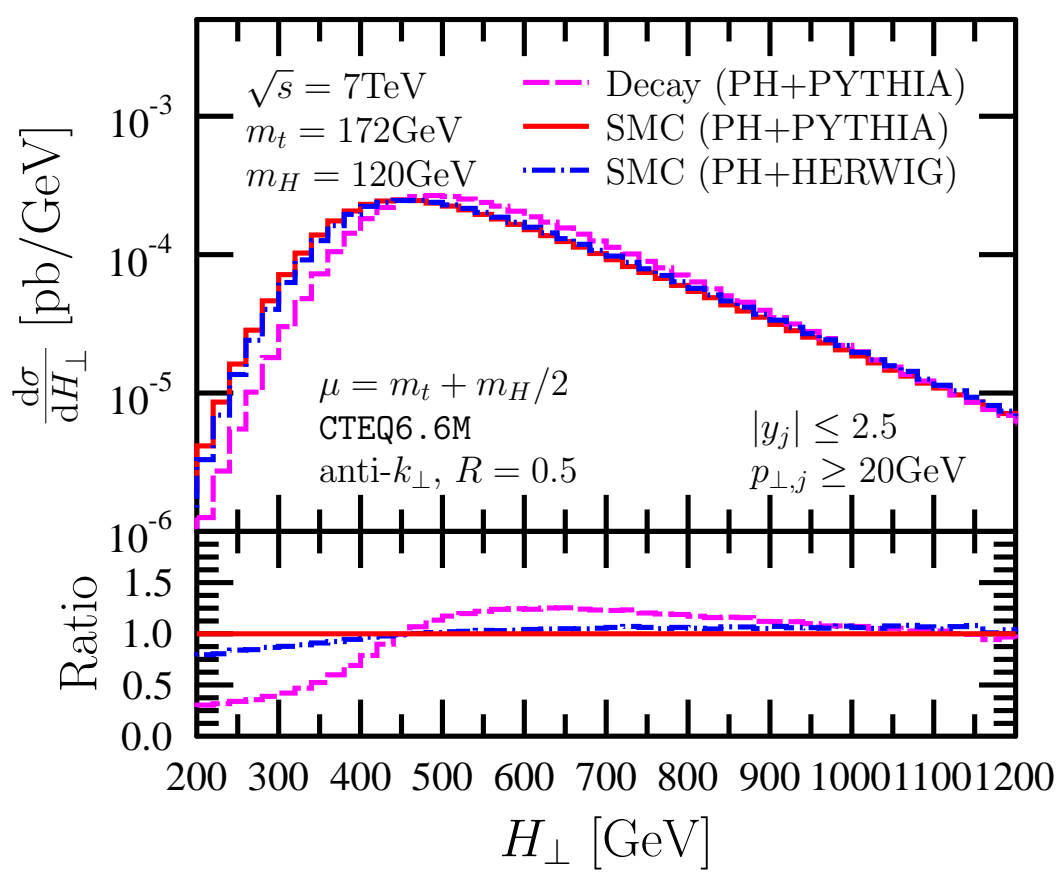

Figure 6: Distribution of the sum of the transverse momenta of all particles, at the decay level (dashed line) and at the SMC level, as obtained by interfacing POWHEG-HELAC to PYTHIA (solid line) and HERWIG (dash-dotted line). Only hadronic cuts were applied.

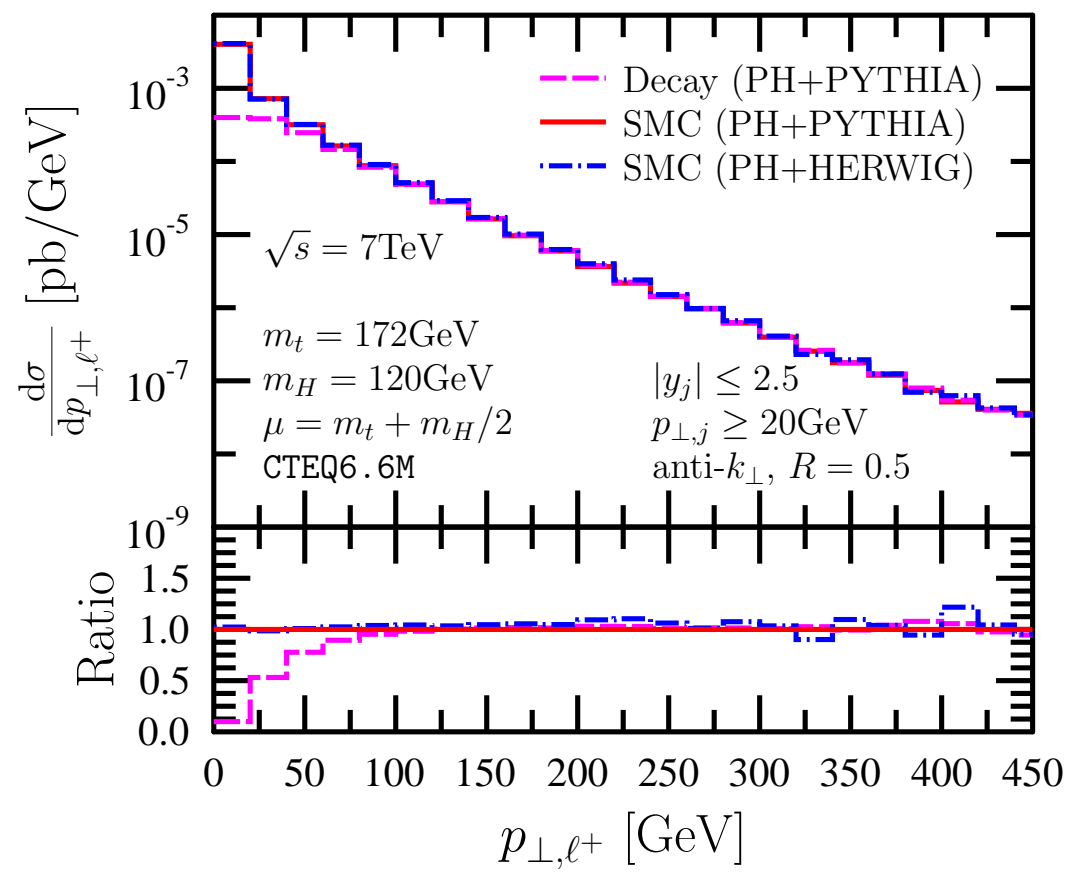

Figure 7: The same as Fig. 6, as for the transverse momentum of all antileptons. 


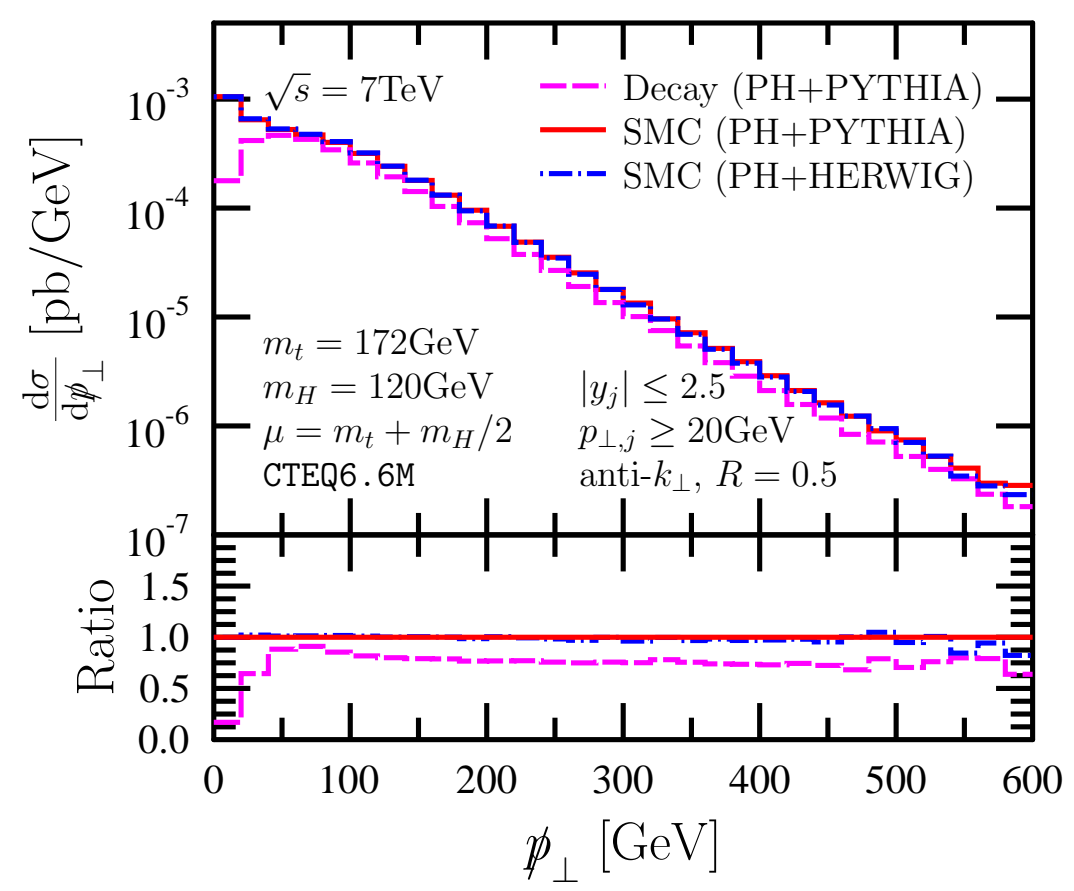

Figure 8: The same as Fig. 6, as for the total missing transverse momentum.

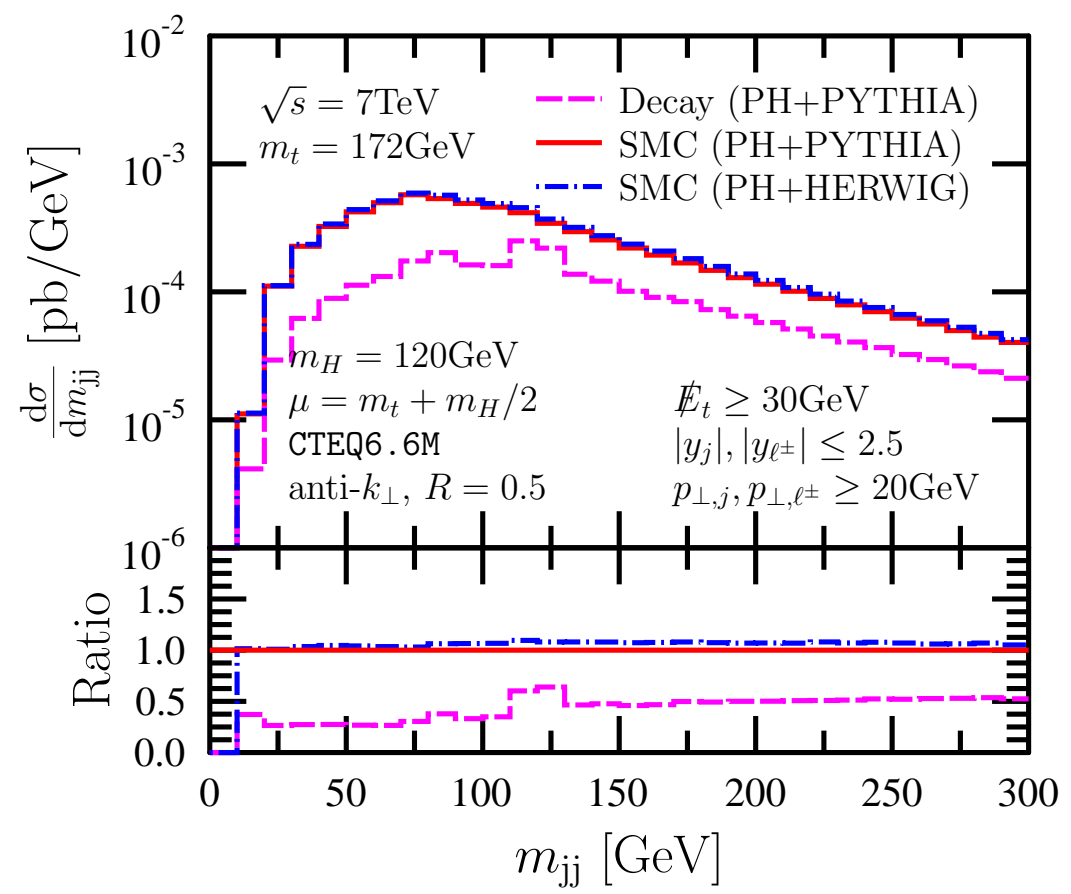

Figure 9: Invariant mass distribution of all jet pairs. Results at the decay level (dashed line) are shown together with results after showering and hadronization, obtained by interfacing POWHEG-HELAC predictions to both the PYTHIA (solid line) and HERWIG (dashdotted line) SMC. The full set of hadronic and leptonic cuts was applied. 
even closer.

\section{Conclusions}

We would like to conclude this letter by emphasizing that interfacing NLO calculations, as structured in HELAC-NLO, with PS and hadronization effects, within the POWHEG framework, open the exciting possibility of realistic, precise and reliable simulations of pp hard-scattering collisions, leading to multiparticle production, up to the hadron level, as detected in the accelerator experiments. We have presented predictions for the signal, $\mathrm{pp} \rightarrow \mathrm{t} \overline{\mathrm{t}} \mathrm{H}$ production, illustrating their consistency with existing calculations, as well as the phenomenological potential of our approach. A thorough analysis, including the most important backgrounds, notably pp $\rightarrow$ t $\bar{t} b \bar{b}$, within the same framework, is in progress and will be reported elsewhere. The event files produced by POWHEG-HELAC, together with further detail and results of our project, are available at http://grid.kfki.hu/twiki/bin/view/DbTheory/TthProd.

\section{Acknowledgements}

This research was supported by the HEPTOOLS EU program MRTN-CT-2006-035505, the LHCPhenoNet network PITN-GA-2010-264564, the Swiss National Science Foundation Joint Research Project SCOPES IZ73Z0_1/28079, the MEC project FPA 2008-02984 (FALCON), and the TÁMOP 4.2.1./B-09/1/KONV-2010-0007 project. We are grateful to M. Krämer for providing us the data files with their NLO predictions [26]. M.V.G. is grateful to the Departamento de Física Teórica y del Cosmos of Granada University for hospitality. A.K. is grateful to G. Bevilacqua for useful discussions on the original HELAC programs.

\section{References}

[1] Frixione, S., And WebBer, B. R. Matching NLO QCD computations and parton shower simulations. JHEP 06 (2002), 029, hep-ph/0204244.

[2] Frixione, S., Stoeckli, F., Torrielli, P., Webber, B. R., And White, C. D. The MCaNLO 4.0 Event Generator. 1010.0819[hep-ph].

[3] Nason, P. A new method for combining NLO QCD with shower Monte Carlo algorithms. JHEP 11 (2004), 040, hep-ph/0409146.

[4] Frixione, S., Nason, P., And Oleari, C. Matching NlO QCD computations with Parton Shower simulations: the POWHEG method. JHEP 11 (2007), 070, 0709.2092[hep-ph].

[5] Bevilacqua, G., Czakon, M., Garzelli, M. V., van Hameren, A., Malamos, I., Papadopoulos, C. G., Pittau, R. and Worek, M. NLO QCD calculations with HELAC-NLO. Nucl. Phys. Proc. Suppl. 205-206 (2010), 211-217, 1007.4918[hep-ph]. 
[6] Kardos, A., Papadopoulos, C., And Trocsanyi, Z. Top quark pair production in association with a jet with NLO parton showering. 1101.2672[hep-ph].

[7] Dittmaier, S., ET AL. Handbook of LHC Higgs Cross Sections: 1. Inclusive Observables. 1101.0593[hep-ph].

[8] Frederix, R., ET AL. Scalar and pseudoscalar Higgs production in association with a top-antitop pair. Phys. Lett. B701 (2011), 427-433, 1104.5613[hep-ph].

[9] Alioli, S., Nason, P., Oleari, C., And Re, E. A general framework for implementing NLO calculations in shower Monte Carlo programs: the POWHEG BOX. JHEP 06 (2010), 043, 1002.2581[hep-ph].

[10] Alioli, S., Hamilton, K., Nason, P., Oleari, C., and Re, E. Jet pair production in POWHEG. JHEP 04 (2011), 081, 1012.3380[hep-ph].

[11] Melia, T., Nason, P., Rontsch, R., and Zanderighi, G. W+W+ plus dijet production in the POWHEGBOX. Eur. Phys. J. C71 (2011), 1670, 1102.4846[hep$\mathrm{ph}]$.

[12] Oleari, C., And Reina, L. W b bbar production in POWHEG. 1105.4488[hep$\mathrm{ph}$.

[13] Melia, T., Nason, P., Rontsch, R., and Zanderighi, G. W+W-, WZ and ZZ production in the POWHEG BOX. 1107.5051[hep-ph].

[14] van Hameren, A., Papadopoulos, C. G., and Pittau, R. Automated oneloop calculations: a proof of concept. JHEP 09 (2009), 106, 0903.4665[hep-ph].

[15] Cafarella, A., Papadopoulos, C. G., and Worek, M. Helac-Phegas: a generator for all parton level processes. Comput. Phys. Commun. 180 (2009), 19411955, 0710.2427[hep-ph].

[16] Czakon, M., Papadopoulos, C. G., and Worek, M. Polarizing the Dipoles. JHEP 08 (2009), 085, 0905.0883[hep-ph].

[17] Ossola, G., Papadopoulos, C. G., and Pittau, R. Reducing full one-loop amplitudes to scalar integrals at the integrand level. Nucl. Phys. B763 (2007), 147169, hep-ph/0609007.

[18] Draggiotis, P., Garzelli, M. V., Papadopoulos, C. G., and Pittau, R. Feynman Rules for the Rational Part of the QCD 1-loop amplitudes. JHEP 04 (2009), 072, 0903.0356[hep-ph].

[19] Alwall, J., Et Al. A standard format for Les Houches event files. Comput. Phys. Commun. 176 (2007), 300-304, hep-ph/0609017.

[20] Suostrand, T., Eden, P., Friberg, C., Lonnblad, L., Miu, G., Mrenna S., Norrbin, E. High-energy physics event generation with PYTHIA 6.1. Comput. Phys. Commun. 135 (2001), 238-259, hep-ph/0010017. 
[21] Sjostrand, T., Mrenna, S., And Skands, P. Z. PYTHiA 6.4 Physics and Manual. JHEP 05 (2006), 026, hep-ph/0603175.

[22] Corcella, G., Knowles, I. G., Marchesini, G., Moretti, S., Odagiri, K., Richardson, P., Seymour, M. H., Webber, B. R. HeRWiG 6.5: an event generator for Hadron Emission Reactions With Interfering Gluons (including supersymmetric processes). JHEP 01 (2001), 010, hep-ph/0011363.

[23] Corcella, G., Knowles, I. G., Marchesini, G., Moretti, S., Odagiri, K., Richardson, P., Seymour, M. H., Webber, B. R. HERWIG 6.5 release note. hep-ph/0210213.

[24] Latunde-Dada, O., Gieseke, S., and Webber, B. A positive-weight next-toleading-order Monte Carlo for e+ e- annihilation to hadrons. JHEP 02 (2007), 051, hep-ph/0612281.

[25] Beenakker, W., Dittmaier, S., Kramer, M., Plumper, M., Spira, M., ZERWAS, P. M. Higgs radiation off top quarks at the Tevatron and the LHC. Phys. Rev. Lett. 87 (2001), 201805, hep-ph/0107081.

[26] Beenakker, W., Dittmaier, S., Kramer, M., Plumper, M., Spira, M., ZERWAS, P. M. NLO QCD corrections to t anti-t H production in hadron collisions. Nucl. Phys. B653 (2003), 151-203, hep-ph/0211352.

[27] Reina, L., AND Dawson, S. Next-to-leading order results for t anti-t h production at the Tevatron. Phys. Rev. Lett. 87 (2001), 201804, hep-ph/0107101.

[28] Reina, L., Dawson, S., And Wackeroth, D. QCD corrections to associated $\mathrm{t}$ anti-t $\mathrm{h}$ production at the Tevatron. Phys. Rev. D65 (2002), 053017, hepph/0109066.

[29] Dawson, S., Jackson, C., Orr, L. H., Reina, L., and Wackeroth, D. Associated Higgs production with top quarks at the Large Hadron Collider: NLO QCD corrections. Phys. Rev. D68 (2003), 034022, hep-ph/0305087.

[30] Hirschi, V., ET AL. Automation of one-loop QCD corrections. JHEP 05 (2011), 044, 1103.0621[hep-ph].

[31] Frederix, R. private communication.

[32] Martin, A. D., Roberts, R. G., Stirling, W. J., And Thorne, R. S. Uncertainties of predictions from parton distributions. 1: Experimental errors. Eur. Phys. J. C28 (2003), 455-473, hep-ph/0211080.

[33] Whalley, M. R., Bourilkov, D., and Group, R. C. The Les Houches Accord PDFs (LHAPDF) and Lhaglue. hep-ph/0508110.

[34] Cacciari, M., Salam, G. P., And Soyez, G. The anti-kt jet clustering algorithm. JHEP 04 (2008), 063, 0802.1189[hep-ph].

[35] Andersen, J., ET AL. The SM and NLO multileg working group: Summary report. 1003.1241[hep-ph]. 